\title{
Polydatin, a natural precursor of resveratrol, induces cell cycle arrest and differentiation of human colorectal Caco-2 cell
}

Salvatore De Maria ${ }^{1,2+}$, Ilaria Scognamiglio ${ }^{3 \dagger}$, Angela Lombardi ${ }^{3}$, Nicola Amodio ${ }^{4}$, Michele Caraglia ${ }^{3}$, Maria Cartenì ${ }^{1}$, Gianpietro Ravagnan ${ }^{1,5}$ and Paola Stiuso ${ }^{3^{*}}$

\begin{abstract}
Background: Human colon adenocarcinoma cells are resistant to chemotherapeutic agents, such as anthracyclines, that induce death by increasing the reactive oxygen species. A number of studies have been focused on chemo-preventive use of resveratrol as antioxidant against cardiovascular diseases, aging and cancer. While resveratrol cytotoxic action was due to its pro-oxidant properties. In this study, we investigate whether the Resveratrol (trans-3,5,49-trihydroxystilbene) and its natural precursor Polydatin (resveratrol-3-O-b-mono- D-glucoside, the glycoside form of resveratrol) combination, might have a cooperative antitumor effect on either growing or differentiated human adenocarcinoma colon cancer cells.

Methods: The polydatin and resveratrol pharmacological interaction was evaluated in vitro on growing and differentiated Caco-2 cell lines by median drug effect analysis calculating a combination index with CalcuSyn software. We have selected a synergistic combination and we have evaluated its effect on the biological and molecular mechanisms of cell death.
\end{abstract}

Results: Simultaneous exposure to polydatin and resveratrol produced synergistic antiproliferative effects compared with single compound treatment. We demonstrated that polydatin alone or in combination with resveratrol at 3:1 molar ratio synergistically modulated oxidative stress, cell cycle, differentiation and apoptosis. Worthy of note treatment with polydatin induced a nuclear localization and decreased expression of heat shock protein 27, and vimentin redistributed within the cell.

Conclusions: From morphological, and biochemical outcome we obtained evidences that polydatin induced a transition from a proliferative morphology to cell-specific differentiated structures and caused human CaCo-2 cell death by induction of apoptosis. Our data suggest the potential use of polydatin in combination chemotherapy for human colon cancer.

Keywords: Human colon carcinoma, hsp27, Differentiation

\section{Introduction}

Colorectal cancer $(\mathrm{CRC})$ remains a leading cause of mortality among many racial and ethnic groups throughout the world [1-3]. Adenocarcinoma cells, such as CRC cells, are remarkably resistant to damage induced by radiation or systemic, immunological and chemotherapeutic agents. As a consequence, the tumours are hard to treat and often

\footnotetext{
* Correspondence: paola.stiuso@unina2.it

${ }^{\dagger}$ Equal contributors

${ }^{3}$ Department of Biochemistry, Biophisics and General Pathology, Second University of Naples, Naples, Italy

Full list of author information is available at the end of the article
}

proliferate rapidly, even under conditions that may adversely affect normal cells. The mechanisms underlying its survival advantage may be related in part to the high endogenous expression of stress proteins. In contrast to normal cells, the basal level of inducible heat shock proteins (HSPs) are frequently higher in tumour cells $[4,5]$ Phytochemicals are among the most promising chemopreventive and treatment options for the management of cancer. The ideal characteristics or chemopreventive/therapeutic agents is the specific modulation of aberrant signalling pathways through the induction of

\section{Biomed Central}


apoptosis [6]. Resveratrol (trans-3,5,49-trihydroxystilbene) and its natural precursor Polydatin (resveratrol-3-O-bmono- D-glucoside Product Origin: Root of Polygonum cuspidatum) are phytoalexins, molecules produced by spermatophytes plants to protect germinal centers, fruits, and roots, by attack by fungi, bacteria or free radicals [7]. Chemically these molecules are stilbenes derivatives. In the Resveratrol there are 3 hydroxyl-groups in 3, 4, and 5 position of stilbene scaffold whereas Polydatin has the position 3 is occupied by a glucopiranoside ring. Substitution of position 3 with a sugar molecule do as not interfere with scavenger functions of the hydroxi-stilbene (ISBs) that is ascribed in major measure to $4^{\prime} \mathrm{OH}$. Wallerath et al. [8] reported that resveratrol might rapidly increase NO production in cultured endothelial cells. At physiologic concentrations, NO protects the gastrointestinal mucosa from injury. By acting as an antioxidant, inhibiting leukocyte adherence, and maintaining mucosal blood flow $[9,10]$. During inflammation, intestinal epithelial cells are exposed to cytokines, bacterial products, and many other substances that affect cellular functions [11]. Under these conditions, NO synthase II is induced in a variety of cells including the intestinal epithelium. NO synthase II, is capable of generating high local intracellular and extracellular concentrations of NO [12-14]. As part of the inflammatory process or sepsis, activated inflammatory cells generated large amounts of superoxide anions $\left(\mathrm{O}_{2}{ }^{-}\right)$. Abundant $\mathrm{NO}$ and $\mathrm{O}_{2}{ }^{-}$radicals react rapidly to form peroxynitrite $\left(\mathrm{ONOO}^{-}\right)$, an extremely reactive and toxic molecule [15-17]. Peroxynitrite is capable of nitrating the tyrosine residues of proteins, thereby disrupting cellular signalling systems that depend upon tyrosine phosphorylation $[18,19]$. Dietary polyphenols with phenol rings are metabolized by peroxidase to form prooxidant phenoxy radicals which are sufficiently reactive to co-oxidize GSH or NADH accompanied by extensive oxygen uptake and ROS formation. The aim of this work is to investigate the in vitro antioxidation and antiproliferative effects of polydatin and resveratrol alone or in combination in human colon adenocarcinoma $\mathrm{CaCo}-2$ cells. This cell line is not particularly sensitive to treatment with chemotherapeutic agents, that induce death by oxidative stress. Moreover, as differentiated Caco2 cells is a well-accepted model for human enterocytes, they have been used to characterize a safety profile of compounds in terms of cell selectivity [20,21]. Moreover, we investigated the effects of polydatin and resveratrol and its combination on colon adenocarcinoma cell lines in terms of growth and apoptosis, cell cycle differentiation and modulation of HSP27, iNOS and vimentin intracellular level and distribution.

\section{Material and methods Chemicals}

All chemicals, of the highest available quality, were obtained from Sigma Chemical Co. (St. Louis, USA). Trans-polydatin and trans-resveratrol, with a purity grade higher than 99\%, have been supplied by Ghimas spa (Casalecchio, Bologna, Italy). The compounds were prepared in according to the method described in patent EP 1292319 B1 and EP 1292320 [22,23].

\section{Cell culture}

Caco-2 (American Type Culture Collection, Rockville, MD, USA), was grown at $37^{\circ} \mathrm{C}$ in h-glucose MEM containing: $1 \%$ (by vol) non-essential amino acids and supplemented with $10 \%$ (by vol) de-complemented fetal bovine serum (FBS) (Flow, McLean, VA, USA), $100 \mathrm{U} \cdot \mathrm{mL}-1$ penicillin, $100 \mathrm{mg} \cdot \mathrm{mL}-1$ streptomycin, $1 \% \mathrm{~L}$-glutamine and $1 \%$ sodium pyruvate. Cells were grown (17-21 passages) in a humidified atmosphere of $95 \%$ air $/ 5 \% \mathrm{CO} 2$ at $37^{\circ} \mathrm{C}$, and in six multi-well plates at different cell densities. After incubation for $4 \mathrm{~h}$ in Dulbecco's modified Eagle's medium (DMEM) with $10 \% \mathrm{FBS}$, the cells were washed with $1 \%$ phosphate-buffered saline (PBS) to remove unattached dead cells, and were incubated with different concentrations of trans-resveratrol (trans-3,5,4'-trihydroxystilbene) and trans-polydatin (trans-5,4'-dihydroxystilbene-3-O- $\beta$ D-glucopyranoside). All experiments were performed in triplicate.

\section{Sensitivity of the cell lines to hydroxystilbenes (ISBn)}

We assessed the sensitivity of the cell lines tested to ISBn using a microplate colorimetric assay that measures the ability of viable cells to transform a soluble tetrazolium salt (MTT) to an insoluble purple formazan precipitate. Cells were plated at the appropriate density $\left(5 \times 10^{3}\right.$ undifferentiated Caco- 2 cells per well and $20 \times 10^{3}$ differentiated Caco-2 cells per well) in 96-well microtitre plates. After $4 \mathrm{~h}$, cells were exposed to various concentrations of ISBn for $24 \mathrm{~h}$. Then, $50 \mu \mathrm{L}$ of MTT $(1 \mathrm{mg} \cdot \mathrm{mL}-1)$ and $200 \mu \mathrm{L}$ of medium were added to the cells in each well. After a $4 \mathrm{~h}$ incubation at $37^{\circ} \mathrm{C}$, the medium was removed, then the formazan crystals were solubilized by adding 150 $\mu \mathrm{L}$ of DMSO and by mixing it in an orbital shaker for 5 min. Absorbance at $550 \mathrm{~nm}$ was measured using a plate reader. Experiments were performed in triplicate. As a control, $0.5 \%$ DMSO was added to untreated cells.

\section{ISBn combination studies}

For the study of the synergism between trans-polydatin and trans - resveratrol on growth inhibition of Caco- 2 cells, the cells were seeded in 96-multiwell plates at the appropriate density $\left(5 \times 10^{3}\right.$ undifferentiated Caco- 2 cells per well and $20 \times 10^{3}$ differentiated Caco- 2 cells per well). After $24 \mathrm{~h}$ incubation at $37^{\circ} \mathrm{C}$ the cells were treated with different concentrations of polydatin (from 0 to $500 \mu \mathrm{M}$ ) or resveratrol (from 0 to $500 \mu \mathrm{M}$ ) and their combinations (50:50, 75:25, 25:75 molar ratio pol:res respectively). Drug combination studies were based on concentration-effect curves 
generated as a plot of the fraction of unaffected (surviving) cells vs. drug concentration after $24 \mathrm{~h}$ of treatment. Assessment of synergy was performed quantitating drug interaction by the Calcusyn computer program (Biosoft, Ferguson, MO). Combination index (CI) values of $<1,1$, and $>1$ indicate synergy, additivity, and antagonism, respectively [15]. Furthermore, we analyzed the specific contribution of trans-polydatin and trans - resveratrol on the cytotoxic effect of the combination by calculating the potentiation factor $(\mathrm{PF})$, defined as the ratio of the IC50 of either trans-polydatin or trans - resveratrol alone to the IC50 of polydatin/resveratrol combinations, respectively, as described before; a higher PF indicates a greater cytotoxicity.

\section{Alkaline phosphatase (ALP) activity}

ALP activity was used as marker of the degree of differentiation of Human CaCo-2 cells. Attached and floating cells were washed and lysed with $0.25 \%$ sodium deoxycholate, essentially as described by Herz et al. [24]. ALP activity was determined using Sigma Diagnostics ALP reagent (no. 245). Total cellular protein content of the samples was determined in a microassay procedure as described by Bradford [25] using the Coomassie protein assay reagent kit (Pierce). ALP activity was calculated as units of activity per milligram of protein.

\section{Nitrite assays}

$\mathrm{NO}$ is rapidly converted into the stable end products nitrite and nitrate. Nitrite was measured by the Griess reaction as reported by Green et al. [26]. The nitrite assay used in this work were described in GomezMonterrey I. et al.

\section{Flow cytometry analysis}

Caco-2 cells were seeded in six multi-well plates at the density of $25 \times 10^{5}$ cells per plate. After $24 \mathrm{~h}$ of incubation with ISBn cells were washed in PBS, centrifuged and directly stained in a propidium iodide (PI) solution $(50 \mathrm{mg}$ PI in $0.1 \%$ sodium citrate, $0.1 \% \mathrm{NP} 40, \mathrm{pH} 7.4$ ) for $30 \mathrm{~min}$ at $4^{\circ} \mathrm{C}$ in the dark. Flow cytometric analysis was performed using a FACScan flow cytometer (Becton Dickinson, San Jose, CA, USA). To evaluate cell cycle, PI fluorescence was collected as FL2 (linear scale) by the ModFIT software (Becton Dickinson). For the evaluation of intracellular DNA content, not less than 20000 events for each point were analysed in at least three separate experiments giving a SD less than $5 \%$. Superoxide anion production in mitochondria was determined by hydroethidine (HE) staining. The treated and untreated cells were incubated for $1 \mathrm{~h}$ with $20 \mathrm{ng} \cdot \mathrm{mL}-1 \mathrm{HE}$, and were scraped, washed twice with PBS and the cell pellet was added to $1 \mathrm{~mL}$ PBS. HE-superoxide anion (HE-O) accumulation was measured by FACScan flow cytometer (FACScan, Becton Dickinson) using CellQuest software. For each sample $2 \times 10^{4}$ events were acquired. Analysis was carried out in triplicate in at least three separate experiments.

\section{Immunostaining and confocal microscopy}

Caco-2 cells grown were fixed in PBS 4\% paraformaldehyde then permeabilized 5 min with PBS 1\% Triton. Immunostaining was carried out by incubation with anti-iNOS, antiHsp27 and anti-vimentin antibodies 1:1000 followed by revelation using Cy3-conjugated anti-rabbit immunoglobulin (Ig) G antibodies (Jackson Immunoresearch Laboratories, West Grove, PA) at a dilution of 1/200 for 45 minutes. The cells were analyzed by an LSM-410 Zeiss confocal microscope.

\section{Evaluation of apoptosis by Western blot analysis}

CaCo- 2 cells were grown for $24 \mathrm{~h}$ with or without Resveratrol and/or polidatin in the previously described experimental conditions. For cell extract preparation, cells were washed twice with ice-cold PBS/BSA, scraped and centrifuged for $30 \mathrm{~min}$ at $4^{\circ} \mathrm{C}$ in $1 \mathrm{ml}$ of lysis buffer ( $1 \%$ Triton, $0.5 \%$ sodium deoxycholate, $0.1 \mathrm{M} \mathrm{NaCl}, 1$ mM EDTA, pH 7.5, $10 \mathrm{mM} \mathrm{Na}_{2} \mathrm{HPO}_{4}, \mathrm{pH} 7.4,10 \mathrm{mM}$ PMSF, $25 \mathrm{mM}$ benzamidin, $1 \mathrm{mM}$ leupeptin, $0.025 \mathrm{U} / \mathrm{ml}$ aprotinin). Equal amounts of cell proteins were separated by SDS-PAGE. The proteins on the gels were electrotransferred to nitrocellulose and reacted with the different MAbs.

\section{Transient transfections}

Cells were seeded at a density of $3 \times 10^{5}$ cells per well in 6 -well dishes ( $\sim 70 \%$ confluency) and transfected in triplicate using the Transfection Reagent according to the manufacturer's protocol as previously described [27].

The pcDNA.3.1- HA-myr-AKT-dominant active construct or the empty pcDNA-em-GFP vectors used in this work were described in Amodio $\mathrm{N}$ et al. [27]. Experiments were repeated at least three times.

\section{Statistical analysis}

Values are expressed as the mean $\pm \mathrm{SE}$. The significance of the difference between the control and each experimental test condition was analysed by unpaired Student's $t$-test, and $\mathrm{P}<0.05$ was considered statistically significant.

\section{Results}

Effect of hydroxy-trans-stilbene (ISBn) in Caco-2 cell lines

We evaluated the effects of both trans-polydatin (Pol) and trans-resveratrol (Res) on the growth inhibition of undifferentiated (exponentially growing) and differentiated (post-confluent) Caco-2 cell lines (Figure 1). Both ISBn induced a dose-dependent growth inhibition at $24 \mathrm{~h}$ with 72 and $192 \mu \mathrm{M}$ (IC50) of Pol in growing and differentiated cells respectively, while the IC50 in growing and 


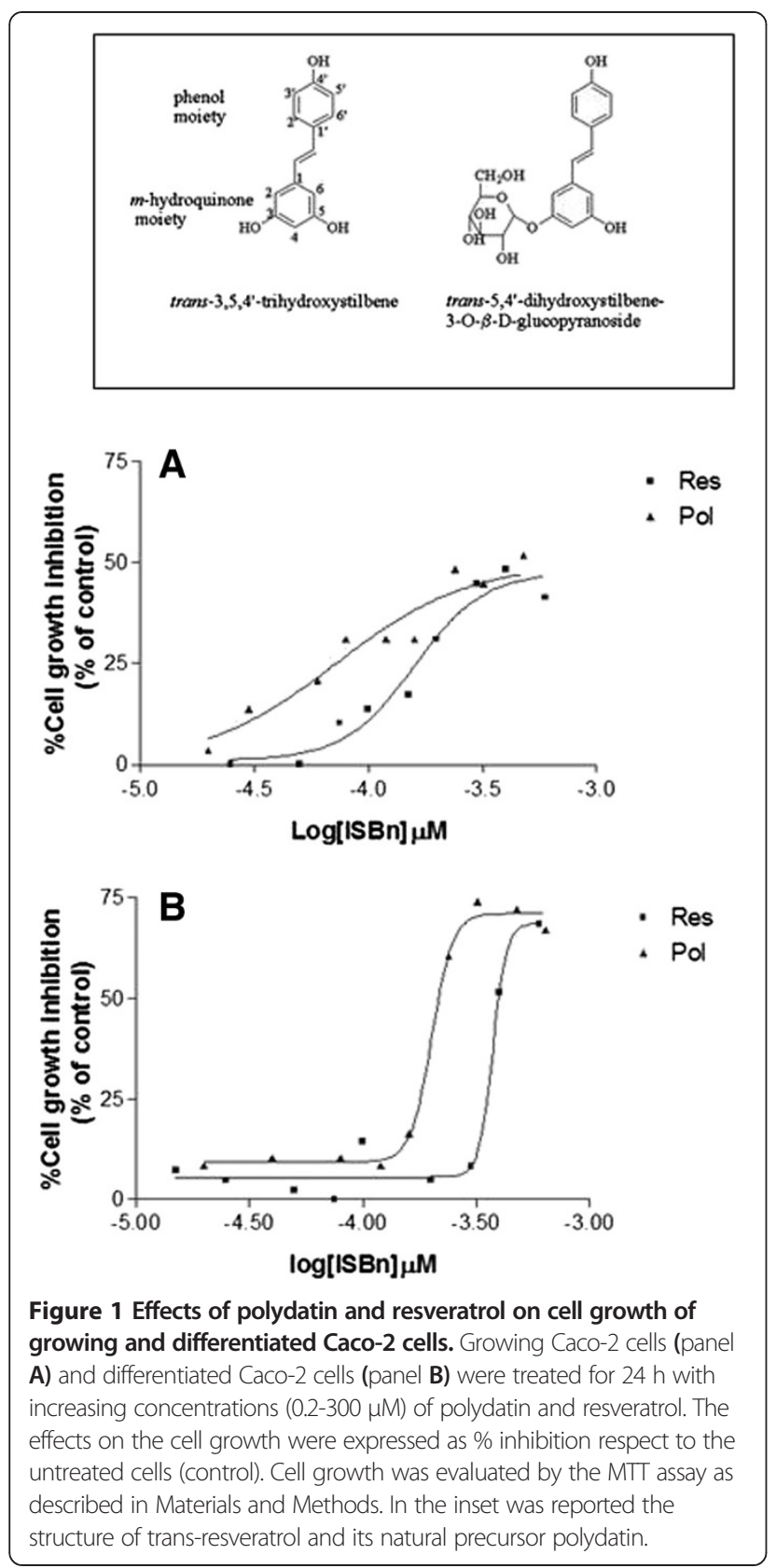

differentiated Caco-2 cells were 156 and $373 \mu \mathrm{M}$, of Res concentrations respectively (Table 1). The antiproliferative effect result higher in the undifferentiated $\mathrm{CaCo}-2$ cells compared to the differentiated cells for both ISBn, moreover the polydatin resulted more cytotoxic respect to resveratrol in both cellular model.

Table 1 Effect of polydatin (Pol), resveratrol (Res), on growth of pre- and post-confluent Caco-2 cells

\begin{tabular}{ccc}
\hline Compound & IC50 $(\boldsymbol{\mu M})$ Pre-conf & IC50 $(\boldsymbol{\mu M})$ Post-conf \\
\hline Res & 156 & 373 \\
Pol & 72 & 192 \\
\hline
\end{tabular}

Synergistic antiproliferative effect of polydatin/resveratrol combination

On the basis of these results, we have evaluated if the ISBn could be synergistic in inducing cell growth inhibition of Caco-2 cells. Specifically, we evaluated the growth inhibition induced by different concentrations of $\mathrm{pol} / \mathrm{res}$ combination at $24 \mathrm{~h}$ on Caco- 2 cell line. We used Calcusyn [28], a dedicated software, to examine the synergism of our treatments. With this software, synergistic conditions occur when the combination index $(\mathrm{CI})$ is $<1.0$. When CI is $<0.5$ the combination is highly synergistic. We found that the combination of Pol and Res was synergistic when the two compounds were used at higher concentrations of polydatin $(\mathrm{pol} / \mathrm{res}$ molar ratio $=3: 1)$ on growing Caco-2 cells (Figure 2A). On the other hand, antagonism was recorded when ratios with higher concentrations of Res were used (data not shown). In synergistic drug combination the CI50 (the combination index calculated for 50\% cell survival by isobologram analysis) was 0.88 . While all $\mathrm{Pol} /$ Res combinations at different molar ratios were highly synergistic on differentiated Caco-2 (Figure 2 panel B). These results demonstrate that the synergism on growing Caco- 2 cells is only when the ratio polydatin/resveratrol is 3:1, whereas in the differentiated Caco-2 a very strong synergism can be recorded on cell proliferation when the concentration of resveratrol is higher. All subsequent evaluations were performed at 240 and 100 $\mu \mathrm{M}$ of Pol and Res respectively (3:1 polydatin/resveratrol molar ratio).

\section{ISBn combination induces cell cycle arrest and differentiation of growing Caco-2 cells}

The effect of ISBn on cell proliferation could be due to their actions on cell cycle and the induction of programmed cell death. In order to explore this possibility, we treated exponentially growing Caco- 2 cells with polydatin and resveratrol alone or in combination and assessed its effects on cell cycle (Figure 3). The cells treated with polydatin alone or in combination with resveratrol for $24 \mathrm{~h}$ showed a $27 \%$ increase in the number of Caco-2 cells in S-phase, while G1 populations decreased. This finding was additionally confirmed by increase of p21 expression in Caco-2 cells treated with ISBn combination. As cell division arrest is one of the prerequisites for cell differentiation [28], we determined the effect of ISBn on Caco-2 differentiation by measuring alkaline phosphatase (ALP) activity as a marker of differentiation into enterocytes correlated to post-confluent phase [29]. In growing $\mathrm{CaCo}-2$ cells both polydatin and resveratrol slightly augment the ALP enzyme activity (Figure 3 panel B). Synergistic effect on ALP activity was recorder in 3:1 $\mathrm{pol} / \mathrm{res}$ molar ratio where a maximum of $30 \%$ of increase was reached, compared to untreated cells. On the other 


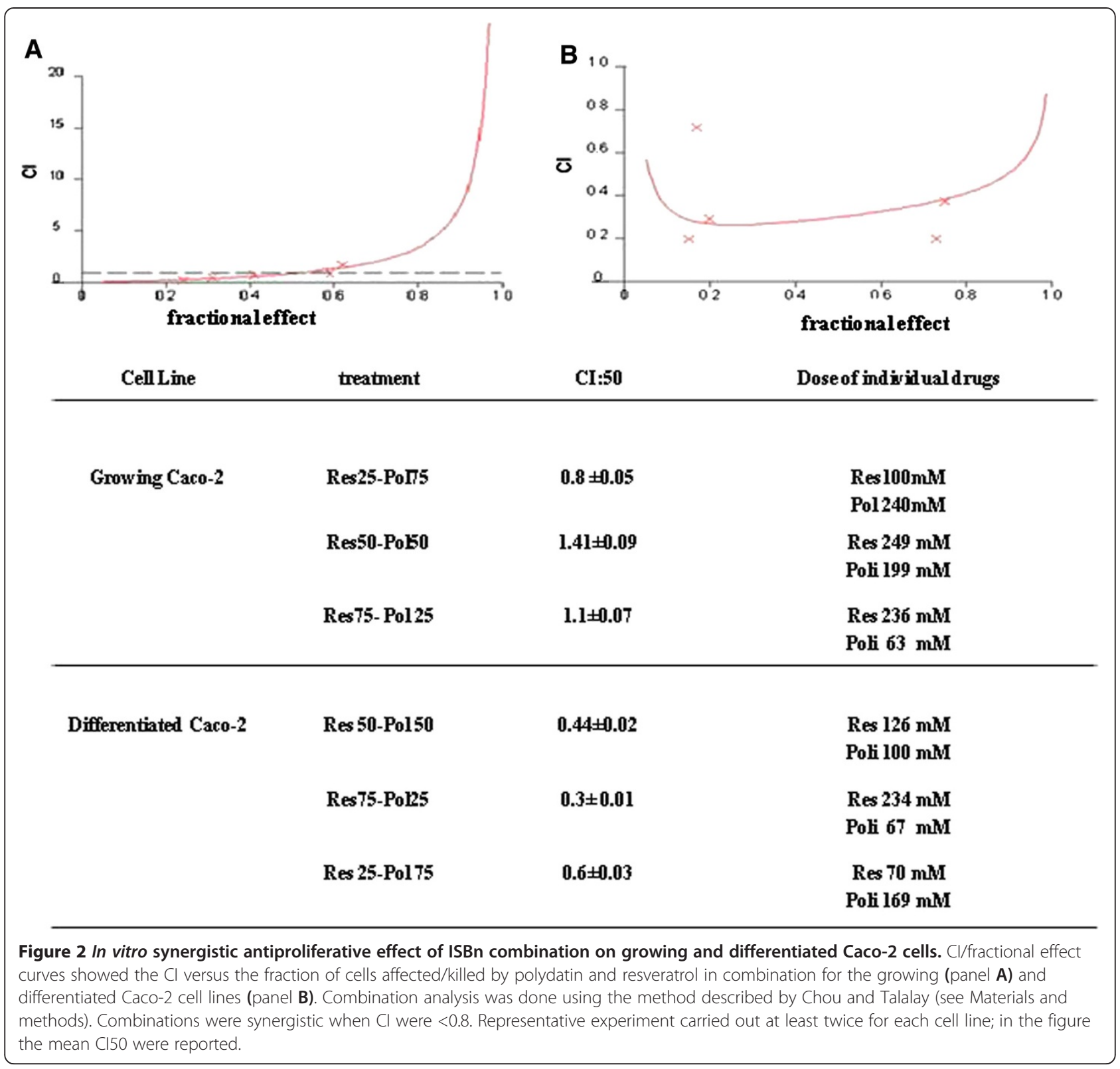

hand, in the differentiated cells 250\%, 70\% and 300\% ALP activity increase with polydatin and resveratrol alone, and ISBn combination treated cells, respectively.

\section{Antioxidant effect of ISBn in CaCo-2 cells}

The antioxidant activity of resveratrol and polydatin alone or in combinations were studied evaluating the mithocondrial superoxide anions, extracellular NO production, and the scavenger enzymes. In Figure 4 were reported the mitochondrial superoxide anions (panel A), in growing and differentiated Caco-2 cells after $24 \mathrm{~h}$ of treatment with Pol, Res and their combination (molar ratio 3:1 Pol/Res). In growing CaCo-2 cells the polydatin determined of 2 fold a reductions of mitochondrial superoxide anions compared to untreated cells (ctr), whereas Res induced a weak increase of superoxide anions. In growing combination treatment Caco- 2 cells the resveratrol mildly antagonized the reduction of superoxide anions induced by polydatin. The ISBs combinations induced a synergistic increase $(p<0.006)$ of TBARS (markers of lipid peroxidation) in the growing Caco-2 cells compared to untreated cells. In differentiated cells polydatin and resvertrol an about 4 and 2fold increase of lipid peroxidatin, respectively, while the ISBn combination treatment induced an additional increase of TBARS values up to 5-fold compared to untreated cells (data not shown). The free NO production was determined in growth medium by measuring 

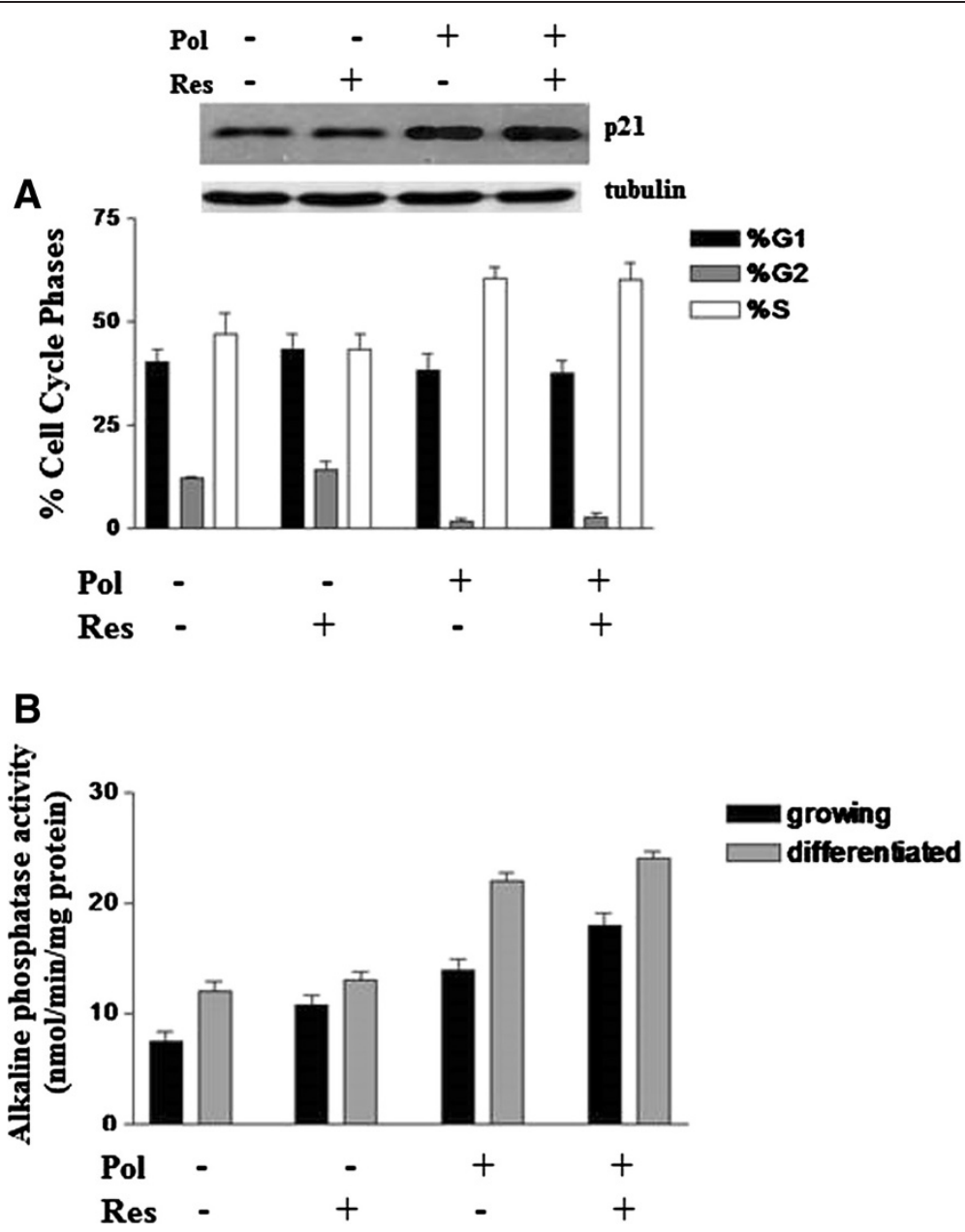

Figure 3 A Effect of ISBn alone or in combinations on the distribution of growing Caco-2 cell populations. Data represent the percentage of cells in each phase of the cell cycle. Cell cycle distribution was determined by DNA flow cytometric analysis. Samples from exponentially growing Caco-2 cells were analysed after $24 \mathrm{~h}$ of treatment with 100 and $240 \mu \mathrm{M}$ of resveratrol and polydatin alone or in combinations. Numbers indicate percentage of cells in G0/G1, S and G2/M phases. Data are representative of four separate analyses. In the inset of panel A was reported the western blot analysis of p21 protein and tubulin expression in growing. B: Effect of ISBn on of Caco-2 cells differentiation. The differentiation of Caco-2 cells assessed by measurement of ALP activity after $24 \mathrm{~h}$ of culture with 0, 240, and $100 \mu \mathrm{M}$ of Pol, Res and their combination. Summary data shown are means $\pm \mathrm{SEM} ;(n=4 ; * P<0.05)$. assessed by measurement of ALP activity.

the stable oxidation products, $\mathrm{NO}_{2}^{-}$. Only the polydatin caused an about 4 and 2-fold increased extracellular $\mathrm{NO}_{2}^{-}$production in growing and differentiated Caco-2 cells respectively (Figure $4 \mathrm{~B}$ ). On the other hand the ISBn combination treatment induced only a slight increase of $\mathrm{NO}_{2}^{-}$if compared to polydatin alone treatment, and this decrease was more evident in growing rather than in differentiated cells (Figure 4 panel B). The treatment of growing cells with ISBn alone or in combination induced a significantly decrease only of catalase activity (Figure 4 panel C). On the other hand, mainly polydatin induced a significantly increased of Mn-SOD activity in differentiated cells (Figure 4 panel D) compared to non treated control cells (CTR).
Polydatin induced differentiation of pre-confluent cells by modulation and cellular localization of iNOS and HSP27 protein

iNOS induction is dramatically increased in postconfluent (differentiated) Caco-2 cells [30]. Level of iNOS expression in pre-confluent $\mathrm{Caco} 2$ cells was low if compared with ISBn-treated cells when analyzed by confocal microscopy (Figure 5 panel A). Polidatin induce iNOS cell localization in the citosol periphery whereas the enzyme was nuclear in resveratrol-treated cells. In growing Caco- 2 cells treated with ISBn combination iNOS was accumulated both periphery and nuclear region. HSP27 immunostaining of preconfluent $\mathrm{Caco} 2$ cells showed an over-expression and cytoplasmic localization with and without resveratrol 

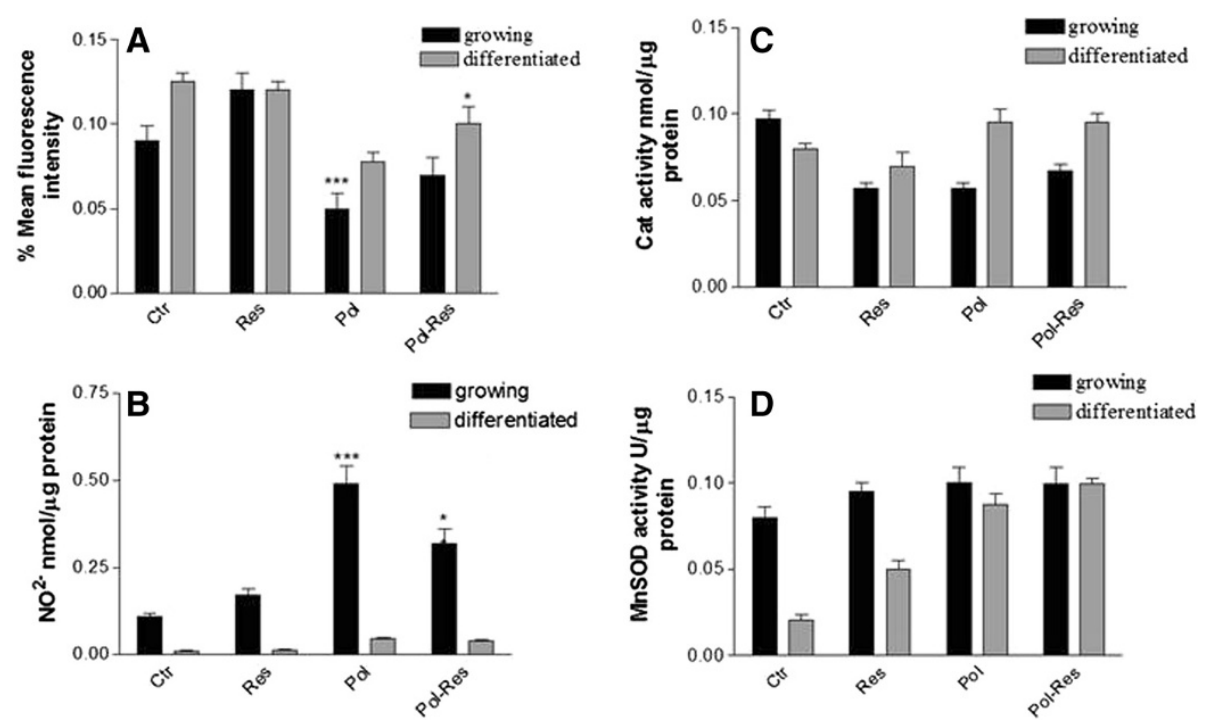

Figure 4 Effect of ISBn alone or in combination on mithocondrial superoxide anions, nitric oxide, and enzyme scavengers activity in Caco-2 cells. (A) After $24 \mathrm{~h}$ Caco-2 treatment with polydatin and resveratrol alone or in combination ( 240 and $100 \mu \mathrm{M})$ at $37^{\circ} \mathrm{C}$, the mitochondrial superoxide anion production was analysed by HE (20 ng · $\mathrm{mL}-1)$ staining. Dye accumulation was analysed by FACScan flow cytometer (FACScan, Becton Dickinson) by the CellQuest software, and the intensities of the bands were expressed as percent control. For each sample, $2 \times 10^{4}$ events were acquired. (B) Nitric oxide was measured in medium. Whole cell homogenates were used for evaluated the Catalase (C) and Mn-SOD activity (D). The bars represent means \pm SEM of three independent experiments. Asterisks indicate significant difference between the Caco-2-treated samples compared with control value ${ }^{* *} P<0.003 ;{ }^{*} P<0.05$.

treatment (Figure 5 panel B). In contrast, polydatin treatment alone or in combination with resveratrol induced a reduces expression and nuclear localization of Hsp27 protein. At the same time, $24 \mathrm{~h}$ treatment with 240 microM polidatin induced a perinuclear redistribution and morphological rearrangement of Vimentin filaments.

The synergistic effect of the ISBn combination on growth inhibition is mediated by apoptosis

Since HSP27 has been shown to inhibit cytochrome c-mediated activation of caspases [31], the apoptotic effect induced by ISB-treatment was investigated by analyzing caspase- 3 activity. Caspase-3 activity was increased in growing Caco-2 cells treated with combination (Figure 5 panel D) [31-33]. In Caco-2 cells grown for $24 \mathrm{~h}$ with Resveratrol and Polydatin we found a marked cleavage of Poly(ADP-ribose) polymerase protein (PARP), paralleled a decrease of the full lengh isoform of the protein (Figure 6). The same data were recorded for caspase 9, a caspase initiator. We have evaluated the effects of resveratrol and polidatin on the terminal enzymes of the survival ras-dependent MAPK pathway, Erk-1 and Erk-2, and p-Erk1 and p-Erk2. We have found that Res alone induced a slight increase of Erk-1 and 2 paralleled a decrease their activities. Pol alone or in combination with Res induced a strong decrease of Erk-1 and Erk-2 expression and a strong increase of their activities both in preconfluent and post confluent cells, as evaluated with a western blotting assay using a mAb raised against the phosphorylated/activated isoforms of the two enzymes. (Figure 6). Thereafter, we have evaluated the effects of the single agents or the combination on another important survival pathway regulated by ras, the Akt/PKB signalling. In details, we have studied Akt expression with western blotting. We have found that Res alone did not induce significant changes in Akt expression while the Pol and the combination caused an almost total reduction of the expression of the enzyme (Figure 6). To demonstrate that polydatin could increase apoptosis by decreasing total AKT protein expression and function, we generated growing Caco-2 cells over-expressing AKT by transiently transfection with a full-length coding sequence of the dominant positive (constitutively activated) AKT gene vector. As shown in Figure 7, both total AKT and phosphorylated Akt were down-regulated in transiently transfected cells only after polydatin treatment. In te same experimental conditions, a concomitant increase of the cleaved form of PARP (Figure 7) was observed. All these data indicate that the synergistic effects of the polydatin and of Res/Pol combination in Caco-2 cells are paralleled by the disruption of two different survival pathways, and an increase of apoptosis.

\section{Discussion}

The epithelium of the small intestine is a highly dynamic continuously renewed system, the whole process of proliferation, differentiation, apoptosis occurs over 3 to 5 days. Cells differentiation process, can be mimed in vitro 

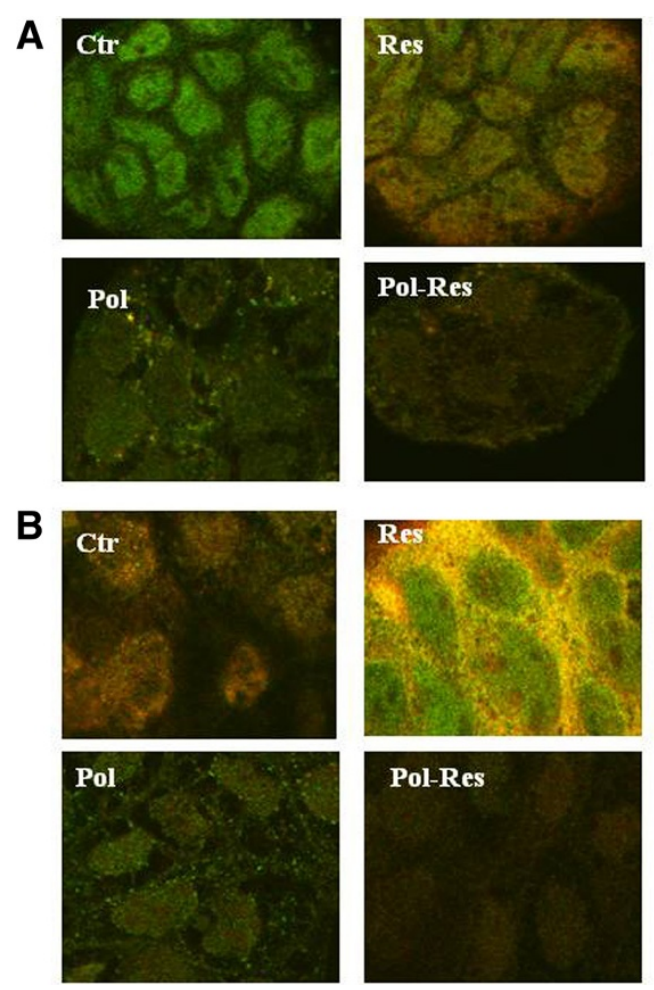

Figure 5 Confocal analysis of ISBn treated growing Caco-2 cells stained for iNOS, hsp27, and vimentin. Panel A: Subcellular co-localization of iNOS (red) and vimentin filaments (green) in Caco-2 cells without ISBn (ctr) and with Pol, Res, and Pol-Res combination treatment. The cells were incubated with anti-vimentin, anti-HSP27 and anti-iNOS antibodies. Images were obtained by confocal microscopy. Panel B: Subcellular co-localization of HSP-27 (red) and vimentin filaments (green) in Caco-2 cells without ISBn (ctr) and with Pol, Res, and Pol-Res combination treatment The cells were incubated with anti-vimentin, anti-HSP27 and anti-iNOS antibodies. Images were obtained by confocal microscopy. Panel C: The expression levels of the proteins are reported as intensities quantified with Image J software. Panel D: Caspase-3 activity on growing and differentiated Caco-2 cells without ISBn (ctr) and with Pol, Res, and Pol-Res combination treatment .Caspase-3 activity was determined by incubating whole-cell extracts with 40 $\mu \mathrm{M}$ caspase-3 substrate and measuring production of hydrolysed 7-amido-4-methyl-coumarin (AMC) groups using a multi-label plate reader. The results are representative of four separate experiments; summary data shown are means \pm SEM.

using Caco-2 cell cultures. Although cancerous in origin, these cells undergo a gradual differentiation process that takes place spontaneously once confluence has been reached. In our previous studies we have investigated the ISBn immune-modulatory [34], anti-inflammatory [35], and anticancer [36-38] properties in melanoma [36], breast cancer [37] and colon cancer [38], derived cellular models. In the present study, we have shown that polydatin, alone or in combination with resveratrol induced cooperative antitumor effect on both growing and differentiated Caco-2 cell lines. In details, cell growth assays showed that the polydatin alone has a stronger cytotoxicity (IC50 $=72 \mu \mathrm{M})$ than Resveratrol $(\mathrm{IC} 50=156 \mu \mathrm{M})$, in growing Caco-2 cells. The synergistic antiproliferative effect was reached only when the poly/res molar ratio was 3:1 as shown by the use of the median effect analysis and calculating CIs, while the combinations at 1 and 0.25 molar ratio were antagonistic. In differentiated Caco-2 cells the IC50 was reached at 192 and $373 \mu \mathrm{M}$ of Pol and Res respectively, and the growth assay clearly showed that all pol/res molar ratio combinations were highly synergistic. The resistance of human colon adenocarcinoma cells to antineoplastic agents, that can induce cell death by oxidative stress are, at least in part related to the high endogenous expression of stress proteins, including the HSP family.

The literature contains extensive documentation for antioxidant (AO) action by resveratrol [39]; however the differential localization of resveratrol and polydatin in the lipid bilayer was also suggested [40] and can give an rationale to explain the synergistic effect observed. There is an apparent contradictions based on an appreciable number of reports providing evidence for pro-oxidant action of Res. Res, acting as AOs for lipids often have a pro-oxidant effect on DNA or protein. The effect of pro-oxidant Res activity, may lead to cell cycle arrest or apoptosis. In our experimental conditions, Res $(100 \mu \mathrm{M})$ elicits pro-oxidant properties as evidenced by an increase of mithocondrial superoxide anion, not paralleled by cell cycle arrest and apoptosis. The Pol, on the other hand, at $240 \mu \mathrm{M}$ induced after $24 \mathrm{~h}$ a significant mithocondrial 


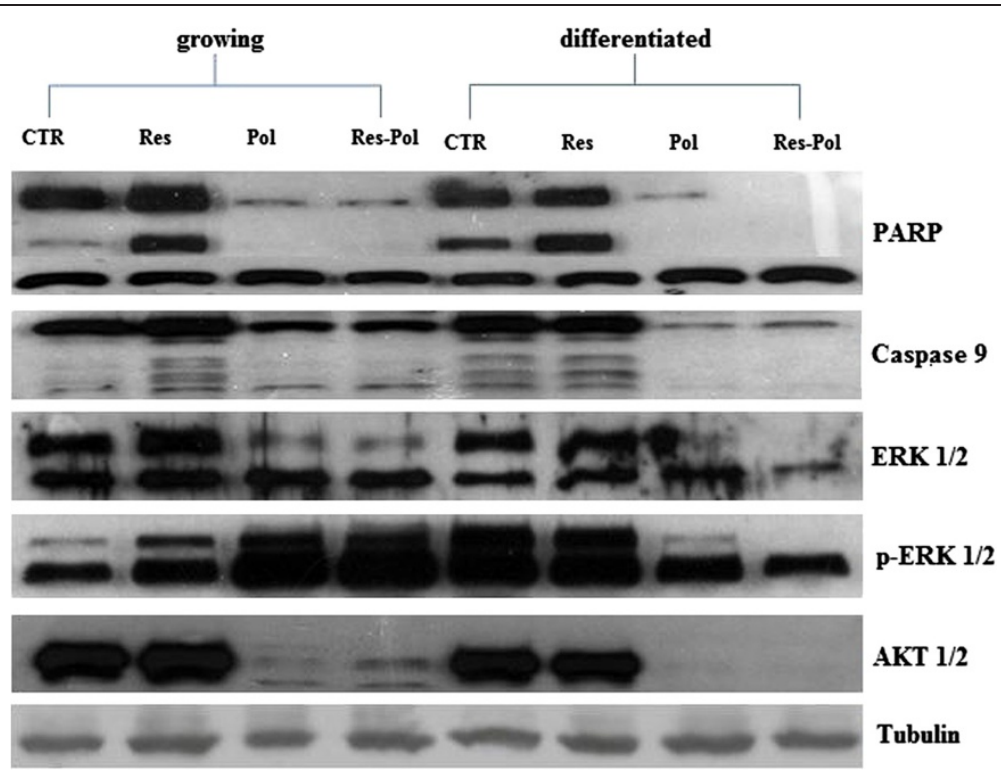

Figure 6 Effects of ISBn treatment on PARP, Caspase 9, total ERK1/2, phosphorylated ERK1/2 (p-ERK), AKT, and tubulin expression evaluated by western blotting. The pre and post-confluences Caco-2 cells were treated for $24 \mathrm{~h}$ with ISBn alone or in combination. The bands associated with expression of PARP, Caspase 9, ERK1/2, phosphorylated ERK1/2 (p-ERK), AKT and house-keeping tubulin, after $24 \mathrm{~h}$ of treatment with ISBn are visualized. The expression of the house-keeping protein tubulin was used as loading control.

superoxide anion reduction especially in growing Caco-2 cells, and this effect is attenuated by combination with Res. The reduction of ROS is not correlated with increased of, manganese superoxide dismutase (MnSOD) activity and was likely be due to direct antioxidant scavenger activity of polydatin. The decreased levels of mitochondrial superoxide anion was paralleled by increase of free NO production, suggesting a potential involvement of $\mathrm{Pol}$ in regulating the balance between $\mathrm{NO}$ and peroxynitrite. A protective effect of free $\mathrm{NO}$ has also been observed in

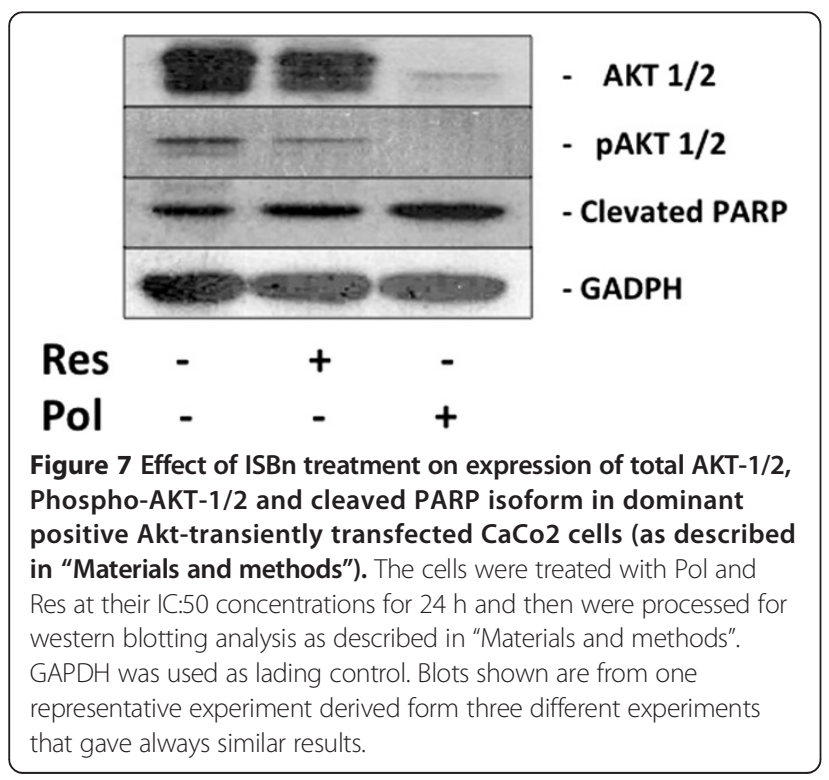

hepatocarcinoma [41], HT-29 human colon carcinoma cell line [42], urinary bladder mucosa [43], and inflammatory cells [44]. Higher level of free NO may be responsible of dynamics of cytoskeleton [45] and cell differentiation [46]. Our finding suggests that the reductions in cell viability in growing Caco- 2 cells elicited by polydatin was also associated to cell structural changes. We observed an increased differentiation evaluated by ALP activity with a concomitant redistribution of Vimentin and HSP27 proteins. The pre-confluent Caco-2 cell population surviving after Pol treatment $(240 \mu \mathrm{M}, 24 \mathrm{~h})$, had a nuclear localization of hsp27, and an increase of cell number in $\mathrm{S}$-phase. All these findings show that polydatin exerts cytotoxic activity through mechanisms of action different from resveratrol. Moreover, it is reasonable to suppose that the cause of polydatin-induced cell death was apoptosis, as suggested by activation of caspase- 3 cystein protease, acting as a common effector pathway for apoptotic processes originating at both cell membrane and mitochondrial levels [47]. In fact we found that polydatin induced apoptosis in Caco- 2 cells as a secondary event, following the modulation of oxidative stress that,in turn induced a redistribution of both HSP27 and vimentin and caused differentiation of Caco- 2 cells into enterocytes, indicating a dose-dependent shift from antioxidant to pro-oxidant effects. However, resveratrol substantially increased iNOS expression. To delineate the mechanisms that underlie the pol-mediated overexpression of $\mathrm{p} 21$, we tested the activation of ERK, that is an important regulators of p21 in other cell lines [47]. In growing Caco-2 cell lines polydatin and 
resveratrol significantly increased ERK1/2 phosphorylation and induced caspase-3 activated apoptosis; these effects, which reached greatest intensity after $24 \mathrm{~h}$ treatment, were paralleled by concomitant reduction in cell numbers. Reductions in cell viability elicited by both these compounds were also associated with cell structural changes, possibly caused by cytoskeleton rearrangements, which could have been responsible for loss of cell survival signals. This implies that cell death was probably preceded by relevant cellular alterations affecting cell survival and proliferation. Moreover, we hypothesized that the cause of ISBs-induced cell death was apoptosis, as suggested by activation of caspase-3, acting as a common effector pathway for apoptotic processes originating at both cell membrane and mitochondrial levels [48]. Promotion of cell death by ERK activation may result from the suppression of survival signalling pathways. The phosphatidylinositol 3-kinase/Akt pathway plays a critical role in the regulation of cell survival, and most growth and survival factors activate this pathway [49]. Recently, it was reported that withdrawal of soluble survival factors from primary cultures of mouse renal proximal tubular cells led to ERK1/2 activation that was accompanied by a gradual decrease in Akt activity and apoptosis [50]. It has been proposed that differentiated Caco-2 cell line may be considered an appropriate model for normal colon cells due to its ability to acquire the phenotype of mature small intestinal cells $[51,52]$. We have found that polydatin has a good profile in terms of cell selectivity, in fact it had a similar and potent cytotoxic activity against the growing Caco-2 cell lines, while it was about 3-fold less potent in differentiated Caco-2 cells. The ability of polydatin to shift the undifferentiated Caco- 2 cells to differentiated enterocytes and then undergo a process of programmed cell death, strongly suggests that this compound should be additionally investigated for its potential use in new combination chemotherapy for colon cancer.

\section{Abbreviations}

NO: Nitric oxide; TBA: 2-thiobarbituric acid; CAT: Catalase; ROS: Reactive oxygen species; SOD: Superoxide dismutase; iNOS: Inducible nitric oxide synthase; HE: Hydroethidine.

\section{Competing interests}

The authors declare that they have no competing interests.

\section{Authors' contributions}

SDM, SI, LA, CM, AN, CM, RGP and PS have critically revised the manuscript and have made substantial contributions to conception. All authors have read and approved the final manuscript.

\section{Author details}

'Operative Unit of Naples GLURES, Academic SPIN-OFF of Ca' Foscari University of Venice, Venice, Italy. Institute "Massimo D'Azeglio", Marano di Napoli, Naples 80016, Italy. ${ }^{3}$ Department of Biochemistry, Biophisics and General Pathology, Second University of Naples, Naples, Italy. ${ }^{4}$ Department of Experimental and Clinical Medicine, Magna Graecia University of Catanzaro, Catanzaro, Italy. ${ }^{5}$ Department of Molecular Sciences and Nanosystems, Ca' Foscari Venezia University, Venice, Italy.
Received: 23 July 2013 Accepted: 8 October 2013

Published: 20 October 2013

\section{References}

1. Bamia C, Lagiou P, Buckland G, Grioni S, Agnoli C, Taylor AJ, Dahm CC, et al: Mediterranean diet and colorectal cancer risk: results from a European cohort. Eur J Epidemiol 2013. [Epub ahead of print] PubMed PMID: 23579425.

2. Hu F, Li D, Wang Y, Yao X, Zhang W, Liang J, Lin C, Ren J, Zhu L, Wu Z, Li S, et al: Novel DNA variants and mutation frequencies of $\mathrm{hMLH} 1$ and $\mathrm{hMSH} 2$ genes in colorectal cancer in the northeast china population. PLoS One 2013, 8(4):e60233. doi: 10.1371/journal.pone.0060233. Epub2013 Apr 3. PubMed PMID: 23573243; PubMed Central PMCID: PMC3616036

3. Zhu C, Bassig BA, Zaridze D, Boyle P, Dai M, Li Q, Zheng T: A birth cohort analysis of the incidence of ascending and descending colon cancer in the United States, 1973-2008. Cancer Causes Control 2013. Epub ahead of print] PubMed PMID: 23535866

4. Jolly C, Morimoto Rl: Role of the heat shock response and molecular chaperones in oncogenesis and cell death. J Nat/ Cancer Inst 2000, 92(19):1564-1572. Review. PubMed PMID: 11018092.

5. Calderwood SK, Khaleque MA, Sawyer DB, Ciocca DR: Heat shock proteins in cancer: chaperones of tumorigenesis. Trends Biochem Sci 2006, 31(3):164-172. Epub 2006 Feb 17. Review. PubMed PMID: 1648378.

6. Manson MM, Farmer PB, Gescher A, Steward WP: Innovative agents in cancer prevention, Recent results in cancer research. Fortschritte der Krebsforschung 2005, 166:257-275.

7. Zamora-Ros R, Andres-Lacueva C, Lamuela-Raventós RM, Berenguer T, Jakszyn P, Martínez C, Sánchez MJ, Navarro C, Chirlaque MD, Tormo MJ, Quirós JR, Amiano P, Dorronsoro M, Larrañaga N, Barricarte A, Ardanaz E, González CA: Concentrations of resveratrol and derivatives in foods and estimation of dietary intake in a spanish population: European prospective investigation into cancer and nutrition (EPIC)-spain cohort. Br J Nutr 2008, 100(1):188-196. Epub 2007 Dec 21. PubMed PMID: 18096094.

8. Wallerath $T$, Deckert $G$, Ternes $T$, Anderson $\mathrm{H}$, Li H, Witte K, Förstermann U: Resveratrol, a polyphenolic phytoalexin present in red wine, enhances expression and activity of endothelial nitric oxide synthase. Circulation 2002, 106(13):1652-1658.

9. Russell J, Okayama N, Alexander JS, Granger DN, Hsia CJ: Pretreatment with polynitroxyl albumin (PNA) inhibits ischemia-reperfusion induced leukocyte-endothelial cell adhesion. Free Radic Biol Med 1998, 25(2):153-159.

10. Thom SR, Bhopale VM, Milovanova TN, Yang M, Bogush M, Buerk DG: Nitric-oxide synthase-2 linkage to focal adhesion kinase in neutrophils influences enzyme activity and $\beta 2$ integrin function. J Biol Chem 2013, 288(7):4810-4818.

11. Magrone T, Jirillo E: The interplay between the gut immune system and microbiota in health and disease: nutraceutical intervention for restoring intestinal homeostasis. Curr Pharm Des 2013, 19(7):1329-1342.

12. Shaked H, Hofseth $L$, Chumanevich A, Chumanevich AA, Wang J, Wang $Y$, Taniguchi K, Guma M, Shenouda S, Clevers H, Harris CC, Karin M: Chronic epithelial NF-KB activation accelerates APC loss and intestinal tumor initiation through iNOS up-regulation. Proc Natl Acad Sci USA 2012 109(35):14007-14012. doi: 10.1073/pnas.1211509109. Epub 2012 Aug 14.

13. Miki K, Kumar A, Yang R, Killeen ME, Delude RL: Extracellular activation of arginase-1 decreases enterocyte inducible nitric oxide synthase activity during systemic inflammation. Am J Physiol Gastrointest Liver Physiol 2009, 297(4):G840-G848. doi: 10.1152/ajpgi.90716.2008. Epub 2009 Aug 27.

14. Keklikoglu N, Koray M, Kocaelli H, Akinci S: iNOS expression in oral and gastrointestinal tract mucosa. Dig Dis Sci 2008, 53(6):1437-1442.

15 Barbieri A, Palma G, Rosati A, Giudice A, Falco A, Petrillo A, Petrillo M, Bimonte S, Di Benedetto M, Esposito G, Stiuso P, Abbruzzese A, Caraglia M, Arra C: " Role of endothelial nitric oxide synthase (eNOS) in chronic stress-promoted tumour growth". J Cell Mol Med 2012, 16(4):920-926. doi: 10.1111/j.1582-4934.2011.01375.x.

16. Washo-Stultz D, Hoglen N, Bernstein H, Bernstein C, Payne CM: Role of nitric oxide and peroxynitrite in bile salt-induced apoptosis: relevance to colon carcinogenesis. Nutr Cancer 1999, 35(2):180-188.

17. Saeki M, Kamisaki Y, Maeda S: Involvement of mitogen-activated protein kinase in peroxynitrite-induced cell death of human neuroblastoma SH-SY5Y cells. Neurosci Res 2000, 38(2):213-216. 
18. Ross GR, Kang M, Akbarali HI: Colonic inflammation alters Src kinase-dependent gating properties of single $\mathrm{Ca} 2+$ channels via tyrosine nitration. Am J Physiol Gastrointest Liver Physiol 2010, 298(6):G976-G984

19. Kusaka G, Uno K, lijima K, Endo H, Asano N, Koike T, Imatani A, Shimosegawa T: The role of nitric oxide in the induction of caudal-type homeobox 2 through epidermal growth factor receptor in the development of Barrett's esophagus. Scand J Gastroenterol 2012, 47(10):1148-1158. Epub 2012 Jul 27.

20. Cotter AA, Jewell C, Cashman KD: The effect of oestrogen and dietary phyto-oestrogens on transepithelial calcium transport in human intestinal-like Caco-2 cells. Br J Nutr 2003, 89(6):755-765.

21. Kamath $\mathrm{AV}$, Darling IM, Morris ME: Choline uptake in human intestinal Caco-2 cells is carrier-mediated. J Nutr 2003, 133(8):2607-2611

22. Ravagnan G, Falchetti R, Lanzilli G, Fuggetta MP, Tricarico M, Mattivi F: Use of extracts from spermatophyte plants with antitumor activity. Pat. EP $12923219 B 1$.

23. Ravagnan G, Falchetti R, Lanzilli G, Fuggetta MP, Tricarico M, Mattivi F: Use of extracts from spermatophyte plants with immunomodulating activity. Pat. EP 1292320

24. Herz F, Schermer A, Halwer M, Bogart LH: Alkaline phosphatase in HT-29, a human colon cancer cell line: influence of sodium butyrate and hyperosmolality. Arch Biochem Biophys 1981, 210(2):581-591.

25. Bradford MM: A rapid and sensitive method for the quantitation of microgram quantities of protein utilizing the principle of protein-dye binding. Anal Biochem 1976, 72:248-254.

26. Green LC, Wagner DA, Glogowski J, Skipper PL, Wishnok JS, Tannenbaum SR: Analysis of nitrate, nitrite, and [15N]nitrate in biological fluids. Anal Biochem 1982, 126(1):131-138.

27. Amodio N, Di Martino MT, Foresta U, et al: miR-29b sensitizes multiple myeloma cells to bortezomib-induced apoptosis through the activation of a feedback loop with the transcription factor Sp1. Cell Death Dis 2012, 3:e436. doi:10.1038/cddis.2012.175.

28. Chou TC, Talalay P: Quantitative analysis of dose effect relationships: the combined effects of multiple drugs or enzyme inhibitors. Adv Enzyme Regul 1984, 22:27-55.

29. Ding QM, Ko TC, Evers BM: Caco-2 intestinal cell differentiation is associated with G1 arrest and suppression of CDK2 and CDK4. Am J Physiol 1998, 275(5 Pt 1):C1193-C1200.

30. Morin MJ, Unno N, Hodin RA, et al: Differential expression of inducible nitric oxide synthase messenger RNA along the longitudinal and crypt-villus axes of the intestine in endotoxemic rats. Crit Care Med 1998, 26:1258-1264.

31. Wink DA, Mitchell JB: Chemical biology of nitric oxide: Insights into regulatory, cytotoxic, and cytoprotective mechanisms of nitric oxide. Free Radic Biol Med 1998, 25(4-5):434-456. Review.

32. Bruey JM, Ducasse C, Bonniaud P, Ravagnan L, Susin SA, et al: Hsp27 negatively regulates cell death by interacting with cytochrome $\mathrm{C}$. Nat Cell Biol 2000, 2:645-652.

33. Bryantsev AL, Loktionova SA, llyinskaya OP, Tararak EM, Kampinga HH, Kabakov AE: Distribution, phosphorylation, and activities of Hsp25 in heat-stressed $\mathrm{H} 9 \mathrm{c} 2$ myoblasts: a functional link to cytoprotection. Cell Stress Chaperones 2002, 7:146-155.

34. Falchetti R, Fuggetta MP, Lanzilli G, Tricarico M, Ravagnan G: Effects of resveratrol on human immune cell function. Life Sci 2001, 70(1):81-96

35. Lanzilli G, Cottarelli A, Nicotera G, Guida S, Ravagnan G, Fuggetta MP: Anti-inflammatory effect of resveratrol and polydatin by in vitro IL-17 modulation. Inflammation 2012, 35(1):240-248.

36. Fuggetta MP, D'Atri S, Lanzilli G, Tricarico M, Cannavò E, Zambruno G, Falchetti R, Ravagnan G: In vitro antitumour activity of resveratrol in human melanoma cells sensitive or resistant to temozolomide. Melanoma Res 2004, 14(3):189-196.

37. Lanzilli G, Fuggetta MP, Tricarico M, Cottarelli A, Serafino A, Falchetti RN, Ravagnan G, Turriziani M, Adamo R, Franzese O, Bonmassar E: Resveratrol down-regulates the growth and telomerase activity of breast cancer cells in vitro. Int J Oncol 2006, 28(3):641-648.

38. Fuggetta MP, Lanzilli G, Tricarico M, Cottarelli A, Falchetti R, Ravagnan G, Bonmassar E: Effect of resveratrol on proliferation and telomerase activity of human colon cancer cells in vitro. J Exp Clin Cancer Res 2006, 25(2):189-193.
39. Kovacic P, Somanathan R: "Multifaceted approach to resveratrol bioactivity Focus on antioxidant action, cell signaling and safety". Oxid Med Cell Longev 2010, 3(2):86-100.

40. Fabris S, Momo F, Ravagnan G, Stevanato R: Antioxidant properties of resveratrol and piceid on lipid peroxidation in micelles and monolamellar liposomes. Biophys Chem 2008, 135(1-3):76-83.

41. Caraglia M, Giuberti G, Marra M, Addeo R, Montella L, Murolo M, Sperlongano P, Vincenzi B, Naviglio S, Prete SD, Abbruzzese A, Stiuso P: "Oxidative stress and ERK1/2 phosphorylation as predictors of outcome in hepatocellular carcinoma patients treated with sorafenib plus octreotide LAR. Cell Death Dis 2006, 20(6):765-773.

42. Wenzel U, Kuntz S, Daniel H: Nitric oxide levels in human preneoplastic colonocytes determine their susceptibility toward antineoplastic agents. Mol Pharmacol 2003, 64(6):1494-1502

43. Andersson MC, Tobin G, Giglio D: Cholinergic nitric oxide release from the urinary bladder mucosa in cyclophosphamide-induced cystitis of the anaesthetized rat. Br J Pharmacol 2008, 153(7):1438-1444. doi:10.1038/bjp.2008.6. Epub 2008 Feb 4.

44. Ronchetti D, Borghi V, Gaitan G, Herrero JF, Impagnatiello F: NCX 2057 2057, a novel NO-releasing derivative of ferulic acid, suppresses inflammatory and nociceptive responses in in vitro and in vivo models. Br J Pharmacol 2009, 158(2):569-579. doi: 10.1111/j.1476-5381.2009.00324.x. Epub 2009 Jul 7

45. Kumarapeli AR, Wang X: Genetic modification of the heart: chaperones and the cytoskeleton. J Mol Cell Cardiol 2004, 37(6):1097-1109.

46. Koti RS, Seifalian AM, McBride AG, Yang W, Davidson BR: The relationship of hepatic tissue oxygenation with nitric oxide metabolism in ischemic preconditioning of the liver. FASEB J 2002, 16(12):1654-1656.

47. Zhu H, Zhang L, Wu S, Teraishi F, Davis J, Jacob D, Fang B: Induction of S-phase arrest and p21 overexpression by a small molecule 2[[3-(2,3-dichlorophenoxy) propyl] amino]ethanol in correlation with activation of ERK. Oncogene 2004, 23:4984-4992.

48. O'Sullivan MP, Tyner JW, Holtzman MJ: Apoptosis in the airways: another balancing act in the epithelial program. Am J Respir Cell Mol Biol 2003, 29(1):3-7.

49. Amaravadi $R$, Thompson CB: The survival kinases Akt and Pim as potential pharmacological targets. J Clin Invest 2005, 115(10):2618-2624.

50. Sinha D, Wang Z, Ruchalski KL, Levine JS, Krishnan S, Lieberthal W, Schwartz JH, Borkan SC: Lithium activates the Wnt and phosphatidylinositol 3-kinase Akt signaling pathways to promote cell survival in the absence of soluble survival factors. Am J Physiol Renal Physiol 2005, 288(4):703-713. Epub 2004 Nov 30.

51. Rousset M: The human colon carcinoma cell lines HT-29 and Caco-2: two in vitro models for the study of intestinal differentiation. Biochimie 1986, 68(9):1035-1040. Review. No abstract available.

52. Stierum R, Gaspari M, Dommels Y, Ouatas T, Pluk H, Jespersen S, Vogels J, Verhoeckx K, Groten J, van Ommen B: Proteome analysis reveals novel proteins associated with proliferation and differentiation of the colorectal cancer cell line Caco-2. Biochim Biophys Acta 2003, 1650(1-2):73-91.

doi:10.1186/1479-5876-11-264

Cite this article as: De Maria et al:: Polydatin, a natural precursor of resveratrol, induces cell cycle arrest and differentiation of human colorectal Caco-2 cell. Journal of Translational Medicine 2013 11:264 